\title{
Les Remarques de Marolles dans ses traductions en prose de l'Énéide : un regard de Moderne sur une œuvre antique?
}

Florence de Caigny

\section{(2) OpenEdition Journals}

Édition électronique

URL : http://journals.openedition.org/rhetorique/884

DOI : $10.4000 /$ rhetorique.884

ISSN : 2270-6909

Éditeur

UGA Éditions/Université Grenoble Alpes

Édition imprimée

ISBN : 978-2-37747-177-5

Référence électronique

Florence de Caigny, « Les Remarques de Marolles dans ses traductions en prose de l'Énéide : un regard de Moderne sur une œuvre antique? », Exercices de rhétorique [En ligne], 13 | 2019, mis en ligne le 06 décembre 2019, consulté le 12 septembre 2020. URL : http://journals.openedition.org/rhetorique/884 ; DOI : https://doi.org/10.4000/rhetorique.884

Ce document a été généré automatiquement le 12 septembre 2020.

\section{c) (i) (2) (2)}

Les contenus de la revue Exercices de rhétorique sont mis à disposition selon les termes de la Licence Creative Commons Attribution - Pas d'Utilisation Commerciale - Partage dans les Mêmes Conditions 4.0 International. 


\title{
Les Remarques de Marolles dans ses traductions en prose de l'Énéide : un regard de Moderne sur une œuvre antique?
}

\author{
Florence de Caigny
}

1 S'il est connu, et parfois décrié par ses contemporains, pour être un traducteur prolifique de poètes latins, l'abbé de Marolles (1600-1681) n'a encore à son actif que la mise en français de Lucain ${ }^{1}$ lorsqu'il publie, en 1649 , une première traduction en prose de l'Énéide, suivie des Bucoliques et des Géorgiques². Durant plus de vingt ans, il n'a de cesse de remettre l'œuvre de Virgile sur le métier. Amendant son premier travail, dont il augmente le volume des Remarques, il fait paraitre une seconde traduction en $1662^{3}$. Puis, délaissant la prose, il offre en 1673 une translation en vers des douze chants de l' Énéide, également assortie de notes ${ }^{4}$. Cette ultime entreprise est le fruit d'un labeur qui s'étend sur plusieurs années et dont on perçoit les étapes intermédiaires dans un volume intitulé l'Énéide de Virgile, traduite en vers par M.D.A.D.V. ${ }^{5}(1671)$. Recueil imprimé à frais d'auteur, cet ouvrage comporte, outre un relevé des comparaisons contenues dans l'Énéide, une traduction partielle ou totale des chants VI, VII, VIII, IX, X et IV, reprise dans l'édition intégrale de $1673^{6}$.

2 L'ensemble de ces traductions est accompagné d'un apparat critique disparate qui prend notamment la forme de commentaires ou de réflexions théoriques sur l'épopée comme en témoigne la présence d'un Traité sur le poème épique inséré dans le volume de 1662. Son abondance et sa variété suggèrent que le projet de Marolles ne s'arrête pas à la seule restitution d'un texte antique et conduisent à s'interroger sur la nature et le but d'un tel travail. En raison de l'ampleur du corpus, nous ne retiendrons ici que les traductions de 1649 et 1662, ensemble dont la cohérence s'articule autour du choix de la prose et des considérations de l'abbé sur l'épopée. Héritier d'une longue tradition de commentaires de l'œuvre virgilienne, Marolles transforme, nous semble-t-il, ce lieu dédié à l'herméneutique en espace de dialogue avec ses contemporains. 
3 L'étude de la figure du destinataire et l'examen des positions théoriques de Marolles sur la traduction nous permettront de saisir l'enjeu initial des Remarques: la mise à disposition fidèle d'un texte antique à un vaste lectorat. Nous verrons ensuite que, dépassant la posture d'interprète-pédagogue, Marolles se fixe comme objectif la lisibilité et la recevabilité de l'Énéide pour des lettrés du XvII siècle. Enfin, un tel projet s'inscrit, à nos yeux, dans celui, plus moderne, d'un dialogue moral, littéraire et politique avec son époque: instrumentalisant l'œuvre de Virgile, l'abbé invite consciemment ou non - à la constitution d'une épopée à la française, par le processus de création littéraire qu'il donne à voir chez les auteurs antiques et contemporains.

\section{L'enjeu des Remarques: mettre à disposition un texte pour le plus grand nombre?}

4 Marolles n'est pas le premier à traduire l'Énéide, comme en témoigne l'énumération des mises en français antérieures à laquelle il procède dans ses Préfaces et en préambule de ses Remarques dans l'une et l'autre de ses éditions. En 1649, il mentionne les traductions partielles de Du Bellay ${ }^{7}$, du Perron ${ }^{8}$, Bertaut ${ }^{9}$, Marie de Gournay ${ }^{10}$ - dont il a été un proche -, de Pierre Perrin ${ }^{11}$ ainsi que d'Annibal Caro dont il signale les versi sciolti ${ }^{12}$. Puis, il évoque les traductions intégrales en vers d'Octavien de Saint Gelais ${ }^{13}$, Louis des Mazures $^{14}$, des frères Robert et Antoine Le Chevalier d'Agneaux ${ }^{15}$, mais également celles en prose de La Mothe du Tertre, qui publie les six premiers livres de Virgile en $1626^{16}$, et de deux autres traducteurs, dont seul l'un est identifié, le Sieur de Tournay de Soissons ${ }^{17}$. En 1662, dans le début de ses notes, il reprend en partie cette liste de traducteurs, qu'il place toutefois après celle des commentateurs - absente de l'édition antérieure - et actualise cette énumération en précisant que Perrin a achevé en 1658 la traduction des six derniers livres de l'Énéide ${ }^{18}$. Cet état des lieux, accompagné dans les Remarques de jugements divers sur la qualité de ces mises en français, plus qu'il n'inscrit le travail de l'abbé dans une succession, permet à ce dernier de spécifier la nature de son entreprise. Celle-ci se veut novatrice en 1649 en ce qu'il est le premier à proposer une version complète qui ne soit pas resserrée «dans les contraintes de la rime, où il est mal-aisé de conserver de bonne grace les pensées d'un Autheur grave \& concis comme Virgile», et il assure les lecteurs qu'ils "y trouveront le vray sens de l'Autheur que [il a] traduit, auquel [il] pense n'avoir rien adjousté ny diminué ${ }^{19}$ ». Il affirme donc avec force le principe d'exactitude, qui ne doit pas faire oublier la recherche de l'élégance dans la formulation, et précise ce que doit être la démarche du traducteur:

[...] j'ay tasché d'y observer jusques aux moindres figures : \& sans estre barbare en nostre langue, j'ay essayé d'y conserver toutes les forces du Latin. Je n'ay fait que suivre, où la phrase a eu du rapport à nostre usage. Mais quand elle luy est contraire, ce qui arrive souvent, il faut inventer, \& se donner quelquefois bien de la peine pour trouver des expressions equivalentes. En cela principalement, si je ne me trompe, consiste l'art de traduire les grands Autheurs : \& je tiens qu'il ne leur faut rien prester, si on ne se veut mettre en hazard de les diminuer : mais aussi ne leur faut-il rien oster, de crainte de les aneantir tout à fait ${ }^{20}$.

Cette double exigence se trouve réaffirmée dans la Préface de 1662 où il revient sur ce qu'il définit comme le «sens » d'un auteur et ses modalités de restitution:

[...] il ne faut pas douter, que la difficulté du sens \& des paroles ne soit beaucoup plus facile à interpreter, qu'il n'est aisé de traduire une periode fort éloquente, \& 
luy conserver en mesme temps sa grace. Sans cela, il est certain qu'il ne se fait point du tout de bonne Version. Et certes, l'élegance \& les belles façons de parler, avec les figures d'Orateur, font bien souvent partie du sens des Illustres Escrivains : car peut-on nier, que s'ils estoient rendus mot à mot, ou qu'on n'y voulust considerer que la force des termes dans leur propre signification, sans se soucier de la grace \& du beau tour, on n'en trahist bien souvent le sens, qui est la plus solide partie de ce que nous lisons ${ }^{21}$ ?

Rendre la force des termes dans leur signification et la beauté de leur formulation, sans perdre ni l'exactitude, ni l'élégance de l'expression dans la restitution : tel est donc le projet que se fixe Marolles dès 1649 et qu'il maintient en $1662^{22}$. Le choix de la prose s'impose alors à lui car il considère qu'elle est la plus adaptée pour réaliser cette gageure et donner accès à l'œuvre au plus grand nombre. Dès le début, il adresse en effet son travail à « ceux qui n'entendent pas assez le Latin, ou qui ne l'entendent point du tout ${ }^{23}$ ", et il ajoute en 1662 qu'il a traduit en prose " pour en donner l'intelligence facile, à plusieurs qui se sont peu souciez des connaissances de la langue latine, ou qui l'ont mal apprise ${ }^{24} »$. Pourtant, il présente, en 1662, une édition bilingue, d'aspect plus érudit par la mise en regard du texte latin avec la traduction et par l'importance des commentaires. Si choisir la prose signifie s'adresser à un destinataire large, quelle(s) fonction(s) confère-t-il alors à cet apparat critique qu'il étoffe ?

6 La liste de quarante et un commentateurs de Virgile, énumérés par Marolles en préambule de ses Remarques ${ }^{25}$, semble établir une filiation entre leur travail et celui de l'abbé. Elle est en réalité une compilation de sources et il n'a probablement consulté que celles qu'il mentionne dans le cours de ces notes (Donat, Servius, La Cerda sont le plus souvent cités). Elle n'en permet pas pour autant de déterminer l'exacte nature ou la fonction des Remarques, sur lesquelles Marolles ne donne que de rares indications dans l'édition de 1662. Dans sa Préface, il signale qu'il a accompagné sa version « de Remarques assez amples ${ }^{26}$ ». De même, avant d'aborder les notes du premier chant, il précise :

Je ne m'estendray que le moins qu'il me sera possible dans les Remarques que je destine à cét Ouvrage : mais, comme il y a beaucoup de choses à observer, je ne voudrois pas répondre aussi qu'elles fussent bien courtes ${ }^{27}$.

7 De fait, Marolles ajoute de nouvelles remarques et amplifie considérablement celles de l'édition de 1649, en insérant notamment ses propres réflexions qui s'appuient sur les traductions qu'il a réalisées entre 1649 et 1662. Il a mis en français Lucrèce en 1650, Horace en 1652, Juvénal, Perse, Catulle et Tibulle en 1653, Properce et de nouveau Lucain en 1654, Martial en 1655, Stace et Plaute en 1658, Lucrèce pour la seconde fois, Térence et Sénèque le Tragique en 1659, il reprend Horace en 1660 et traduit Ovide en 1660-1661. Plusieurs ajouts dans les Remarques de 1662 sont issus de ses analyses sur les poèmes épiques de Stace et Lucain et sont liés à la rédaction du Traité du poème épique, pour l'intelligence de l'Énéide, seul ouvrage théorique de l'abbé de Marolles, joint à la traduction de 1662, comme il le dit explicitement dans la Préface :

lesquelles [remarques] ont du rapport au traité que j'ay fait exprés du Poëme épique sur le modelle de celuy de Virgile, quoy que plusieurs autres en eussent écrit avant moy : Mais ils ne l'ont peust-estre pas fait avec plus de soin, ny avec des Recherches particulières ${ }^{28}$.

Les Remarques apportent certes des éclaircissements sur le genre lui-même, mais le fait de consacrer un ouvrage particulier aux aspects génériques de l'Énéide suggère qu'elles ne s'arrêtent pas à cet aspect. En outre, le souci d'un vaste lectorat est également 
perceptible pour les commentaires (et non simplement pour la traduction) puisqu'il précise, toujours dans la Préface :

J'ay traduit dans mes Remarques tous les passages Latins que j'y ai alleguez. Et pour les Grecs, je n'en ay employé que la Version vulgaire, afin de n'estre pas ennuyeux à ceux qui ne sont pas fort charmez des beautez du Grec \& du Latin, ou qui ont peu de commerce avec l'usage de ces deux langues, qui ne sont vivantes que dans les Livres $^{29}$.

Il n'entend donc pas réserver l'espace des commentaires à un public savant, sans toutefois l'exclure. Pour autant, il ne définit pas la fonction exacte de ces observations.

Un premier parcours des Remarques nous amène à penser qu'elles participent à ce que Marolles appelle «l'intelligence facile» du texte. Leur abondance, sur des aspects autres que génériques ou théoriques, souligne l'échec partiel de la traduction dans la restitution et la compréhension totale du texte, voulues initialement par Marolles; elles apparaissent alors comme un auxiliaire pour la « lisibilité » du texte par le plus grand nombre.

\section{Rendre lisible l'Énéide pour un lecteur du XvII siècle}

9 Rendre lisible le texte de l'Énéide commence par le refus de toute obscurité qui serait liée à une erreur de construction - et dont Marolles aurait pris conscience entre le moment de la rédaction et celui de la réalisation de l'ouvrage - ou liée à une faute typographique lors de l'impression. Les rectifications s'identifient par la présence de la mention «Lisez", tandis que des observations sur le manque de soin de l'imprimeur signalent en plusieurs occasions ce suivi exercé par le traducteur sur la production de ses œuvres, comme en témoigne l'une de ses Remarques du livre X de l'édition de 1649 :

En la ligne 26. Favorable par la fuitte pour où serace. Lisez favorable pour la fuitte, par où sera-ce. Cette faute de l'Imprimeur est demeurée en 500. Exemplaires, \& je l'ay corrigée pour le reste, m'estant heureusement rencontré à l'Imprimerie, comme on tiroit les 1000 . de cette edition ${ }^{30}$.

10 À plusieurs reprises, Marolles fait état de ses scrupules de traducteur : il réaffirme par ce biais sa volonté de fidélité à Virgile et le refus d'une traduction qui serait peu claire pour le lecteur. Les commentaires à l'occasion de passages difficiles en latin sont, sur ce point, significatifs. Marolles y étoffe son propos et justifie ses choix de traduction, en les appuyant sur les annotations des commentateurs antérieurs qu'il cite, confronte, rejette ou suit. Ainsi dans les Remarques du livre IX, commente-t-il un passage du texte virgilien :

Page 22. Euryale prit les ornements \& les ceintures de Ramnes. Ce lieu est difficile \& les interpretes varient au sens \& en la construction des mots. Je pense avoir suivy l'exposition de Servius qui ajouste ces mots en suitte. Sane sciendum hunc locum unum de 12. Virgilis, sive per naturam obscuris, sive insolubilibus, sive emendandis, sive sic relictis ut a nobis per historiae antiquae ignorantiam liquide non intelligantur ${ }^{31}$.

Bien que ne citant pas précisément l'ensemble des commentateurs consultés, Marolles donne à voir que son travail minutieux est guidé par le souci de clarifier le sens du texte.

11 L'obscurité du passage, sans relever de la difficulté de construction latine, peut s'expliquer par le caractère poétique des images utilisées par Virgile. Ainsi, dans les Remarques consacrées au Livre VI précise-t-il à propos du vers 545, et cela dans les deux éditions : 
Page 152. Je suis prest de m'en aller, \& d'achever le nombre.] Il y a au Latin, explebo numerum. Ce qui est mal-aisé à entendre, \& souffre beaucoup d'interprétations. Servius l'explique, je diminueray le nombre, voulant dire qu'il se retireroit de la compagnie d'Enée et de la Sibylle. Mais ceux que j'ay suivis l'entendent, ou du nombre des années qu'il eust deu vivre en ce monde, ou de celles qu'il devoit passer en l'autre dans le lieu de purgation, avant que d'entrer dans le sejour des bienheureux ${ }^{32}$.

L'abbé, se faisant pédagogue, recourt alors à la paraphrase pour expliquer le ou les sens possibles de l'expression.

12 Cette posture est fréquente même lorsque le lecteur n'est pas confronté à une difficulté liée au latin. Le commentateur semble anticiper une éventuelle obscurité en reformulant sa traduction de façon plus commune ; il propose ainsi un synonyme pour le verbe «nettoyer » au livre IX dans les deux commentaires, en l'introduisant par l'expression «c'est à dire » que l'on rencontre quasi systématiquement dans ces cas de figure :

Je nettoiray tout cecy. C'est à dire, je tailleray en pieces tout ce qui se presentera devant moy $^{33}$.

Il signale parfois une figure de style (les prosopopées, beaucoup plus systématiquement les comparaisons ${ }^{34}$ et plus occasionnellement les métaphores), tout en paraphrasant sa traduction quand cela lui paraît nécessaire pour la parfaite intelligence du sens du texte. Ainsi, en 1649, signale-t-il sans plus de développement ce qui lui semble être une prosopopée dans ses Remarques sur le livre VIII :

P. 198. D'où leur vint un grand étonnement.] C'est une sorte de prosopopée, puisque

l'étonnement est attribué aux eaux \& aux forests qui sont des choses insensibles ${ }^{35}$.

L'utilisation de la conjonction de subordination « puisque » introduit la justification de la lecture du passage comme prosopopée : tout en dévoilant les procédés stylistiques dont use Virgile, Marolles en propose indirectement une définition dans l'explication (les eaux et les forêts éprouvent de l'étonnement). Dans l'édition de 1662, il garde cette définition et ajoute des exemples issus d'autres textes qu'il a traduits antérieurement:

92. D'où leur vint un grand étonnement.] C'est une sorte de prosopopée, puisque

l'étonnement est attribué aux eaux \& aux forests qui sont des choses insensibles. Il y a quelque chose de semblable à cecy dans le 9. L. de Stace, où il dit en parlant du fleuve Ismene,

\section{Stupet hospita belli}

Unda viros, claraque armorum incenditur umbra ${ }^{36}$.

Silius dans son 10. L. dit du Tibre, qu'il admira la hardiesse de Clelie qui entreprit de le traverser à la nage, quoy qu'il fust débordé. Et Ovide dans le 2. Livre des Amours, parlant du Navire des Argonautes, écrit que les eaux en furent émerveillées, Mirantibus aequoris undis ${ }^{37}$.

Un tel ajout s'inscrit, pour partie, dans une démarche pédagogique en offrant au lecteur d'autres illustrations du procédé sur une thématique identique.

Enfin, il lui arrive parfois de qualifier la nature d'un passage; ses explications, sans entrer dans les détails, relèvent alors de l'explication du genre du texte et servent à clarifier l'effet recherché par l'auteur. Ainsi au livre IX mentionne-t-il de façon identique dans les deux éditions le début de la plainte de la mère d'Euryale :

Euryale est-ce donc toy ?] C'est le commencement de cette ingénieuse plainte que fait la mere d'Euryale sur la mort de son fils, pour attirer la commiseration de tout le monde, selon toutes les regles de l'art, remarquées par Cicéron au jugement de Servius $^{38}$. 
Il emprunte à ses prédécesseurs une partie de son commentaire mais ne détaille pas, s'arrêtant à la simple mention de " toutes les règles de l'art "; signalant l'effet produit, il célèbre autant l'inventio que l'elocutio de Virgile.

C'est encore en bibliothécaire-pédagogue que Marolles assure le lien entre deux cultures - celle d'une époque révolue et celle du temps présent - en élucidant les allusions du texte qui pourraient échapper à un lecteur non érudit. S'appuyant sur les commentateurs précédents et sur sa propre culture antique - sur ce point, Marolles innove peu -, l'abbé identifie les personnages par leur nom, lorsqu'ils sont désignés par une périphrase ou un adjectif lié à leurs aventures. Pour justifier cette dénomination, il narre ensuite leur histoire et signale la transition de l'identification à l'éclaircissement de la périphrase par l'usage d'expressions telles que "car", "pour ce que ». Cette seconde partie du commentaire est souvent amplifiée dans l'édition de 1662 par la mention d'autres références antiques, qu'il ne cite pas systématiquement dans le texte. De même, pour les lieux géographiques, clarifie-t-il l'appellation et donne-t-il l'origine de cette dernière grâce à des précisions d'ordre historique ou mythologique. Enfin, dans l'établissement de ce lien entre deux époques, il explique certains traits de civilisation : par cette remise en contexte, il permet au lecteur de saisir le sens d'une expression ou d'une situation et d'éviter toute erreur par une lecture anachronique.

Cette démarche du commentateur vise à donner les outils nécessaires à tout lecteur pour saisir pleinement le sens du texte virgilien et à rendre son « intelligence facile». Mais Marolles glisse souvent de la compréhension du sens à sa lisibilité pour un lecteur $\mathrm{du} \mathrm{XVII}^{\mathrm{e}}$ siècle. Il opère à plusieurs reprises des actualisations ou rapprochements avec l'époque contemporaine dans le cadre de ses Remarques. Ils peuvent concerner des aspects pratiques : il justifie par exemple l'emploi du mot français «bourdelier» dans la traduction, terme technique de son époque, pour traduire au vers 45 du livre VIII le latin ubera - qui signifie littéralement "mamelle» - en précisant dans ces deux commentaires que "c'est un terme connu par les chasseurs, pour dire les mamelles de la laye ou de la truye sauvage ${ }^{39} \%$. Les actualisations sont presque systématiques lorsqu'il évoque un lieu géographique pour lequel, outre l'explication mentionnée précédemment, il ajoute la localisation actuelle : cela permet au lecteur de situer le lieu de l'action avec des repères contemporains. Ce souci était visible dès 1649 puisque Marolles, en sus de ses Remarques et de la traduction, propose une «Description du Voyage d'Énée ${ }^{40}$ ", description accompagnée d'une carte pliée et d'une "Explication geographique de tous les lieux mentionnés dans les œuvres de Virgile, par ordre alphabetiques, B. signifie Bucoliques, G. Georgiques, E. Eneide ${ }^{41}$ ».

17 Enfin, cette recherche de lisibilité se perçoit par la confrontation des us et coutumes antiques avec ceux du XVII ${ }^{e}$ siècle. Ainsi, dans ses deux commentaires du Livre VIII de l' Énéide, Marolles suggère une proximité entre les ablutions des Anciens et le baptême chrétien :

Recevez Énée en vostre saincte riviere. Le sujet se presente bien en cet endroit de dire beaucoup de choses des ablutions des Payens : mais pour ne m'esloigner pas de la briesveté que je me suis proposée, il me suffira de remarquer, que par une sagesse incomprehensible, l'esprit de Dieu \& de l'Église, a voulu sanctifier plusieurs coustumes des Gentils, \& qu'il a substitué les eaux incorruptibles du Baptesme, à celles qui estoient mises en usage par la vaine superstition ${ }^{42}$.

73. Recevez Énée en vostre saincte riviere. Le sujet se presente bien en cet endroit de dire beaucoup de choses des ablutions des Payens : mais le sujet en est trop ample : 
\& il me suffit de remarquer, que nostre baptesme n'est pas tant une suitte de cette coustume, qu'elle en est le renouvellement \& la sanctification ${ }^{43}$.

L'exemple concerne ici la religion, mais celle-ci n'est pas le seul domaine d'application de ce type de remarques. Marolles souligne la parenté qu'il peut y avoir entre deux civilisations distantes dans le temps et facilite la réception d'un texte antique profane par le lien établi entre les deux époques. Cependant, l'abbé va souvent au-delà de la simple actualisation par pédagogie et, utilisant le texte de Virgile, il entame dans le cadre de ses Remarques un dialogue avec ses contemporains, dialogue dont les enjeux sont autant moraux que littéraire.

\section{Les Remarques : les enjeux moraux et littéraires d'un dialogue avec son époque}

18 En gommant les distances entre deux civilisations, Marolles opère un glissement: quittant sa position médiane de passeur ${ }^{44}$, il érige l'Énéide en texte édifiant et fait de l'œuvre l'intermédiaire par lequel il dialogue avec ses contemporains. Au détour d'une expression, il propose dans ses Remarques une lecture orientée par son regard d'homme d'Église ou de lettré et qui n'est destinée qu'à l'instruction morale ou la réflexion littéraire.

19 À plusieurs reprises, il souligne en effet la possibilité d'une lecture chrétienne de l'œuvre qui serait profitable et délivre implicitement un enseignement religieux. Ainsi, au Livre II, à propos du vers 777 qu'il traduit par «Ces choses n'arrivent point sans l'ordonnance des Dieux », commente-t-il en 1649 puis en 1662 :

Cette sentence est digne d'un Chrestien, qui doit estre persuadé, que rien n'arrive sans la providence de Dieu ${ }^{45}$.

Il est certain que rien n'arrive sans la Providence de Dieu : Et Creüse ne dit cela que pour consoler Enée qui estoit affligé de sa perte, \& qui avoit trop de pieté pour se plaindre en cette occasion la mesme de la sagesse \& de la Justice des Dieux ${ }^{46}$.

La première "Remarque » marque la filiation entre le héros virgilien et le chrétien, puis l'emploi du verbe «devoir» au présent signale le passage au dogme religieux chrétien. Celle de 1662, supprimant le rapprochement, donne directement le précepte religieux puis propose une explication du texte orientée vers le dévoilement de vertus toutes chrétiennes chez Énée : Marolles, abbé, délivre l'enseignement à tirer pour le lecteur de ce passage. De tels enjeux moraux se trouvaient en filigrane dans la Préface, lorsque Marolles prenait soin de se justifier, en tant qu'ecclésiastique, de traduire des œuvres profanes :

Si quelqu'un me reprend d'avoir travaillé à un ouvrage prophane, au lieu de m'estre occupé à escrire quelques Traittez de piété, ou de Theologie, puis que je suis de profession Ecclesiastique ; encore que je ne m'en veuille pas excuser tout à fait, si est-ce que je ne craindray point de dire, puis qu'il faut rendre compte à Dieu \& au public, de l'employ de toutes ses heures, que non seulement il n'y a rien icy contre les bonnes mœurs, mais encore, que plusieurs y pourront profiter ${ }^{47}$.

Si beaucoup d'analyses de l'homme d'Église concernent des aspects du culte et du dogme chrétien, les plus nombreuses se lisent à propos d'Énée. Cette focalisation s'explique par la lecture politique qu'il donne de l'œuvre de Virgile. Le héros incarne ce n'est pas en soi une nouveauté - la figure du Prince idéal par son courage, sa vertu et sa piété. Marolles ne manque pas de le souligner dans ses Remarques tout en donnant une inflexion contemporaine à ses commentaires : Énée est le modèle auquel peut se référer le monarque français et même plus largement toute personne ayant autorité 
sur le peuple ${ }^{48}$. Une telle orientation s'explique par le contexte dans lequel fut rédigée la première traduction : l'abbé a dû en retarder la publication en raison des troubles de la Fronde qu'il mentionne dans sa Préface. Sa dédicace "AU ROY », alors mineur, n'a rien d'anodin en ce moment de fragilité du pouvoir royal: il établit clairement le parallèle entre le héros de fiction et le jeune Louis XIV, et mentionne pour cela son origine divine qui renvoie à la conception française de la monarchie de droit divin :

Énée qui est du sang des Dieux, représente bien à mon avis, la grandeur de votre extraction, qui tire son origine du plus glorieux Sang de l'Univers ${ }^{49}$.

Lorsqu'il énumère par la suite les qualités attendues d'un souverain, et illustrées par Énée, ce "Prince parfait ${ }^{50}$ ", Marolles n'est pas interprète; il change de posture d'énonciation comme en témoigne l'emploi du verbe «devoir » et de pluriels comme «Les Princes et les Roys » et se fait théoricien de la pratique du pouvoir.

21 L'âge du roi au moment de la première traduction tend à conférer à cette dernière une dimension éducative à destination du Prince, en sus d'un objectif moral pour le plus grand nombre. Elle ne disparaît pas dans la seconde traduction en prose. Certes, Louis XIV n'est plus mineur, mais il vient de prendre les rênes de l'État après la mort de Mazarin en 1661, et le pays sort à peine des agitations de la Fronde. L'ouvrage devient alors une invitation pour le tout jeune prince régnant à conserver les qualités qui font déjà de lui un parangon de monarque. La nouvelle dédicace "AU ROY " en 1662 réaffirme en effet l'identification du héros au monarque mais en soulignant qu'il incarne déjà les qualités que l'on peut attendre d'un roi :

Et Virgile qui n'est jamais inferieur à personne en toutes les choses qu'il écrit, a fait dans son immortel Ouvrage, au sujet de son Heros, une Peinture naive de tout ce que nous voyons en la Personne Royale de Vostre majesté, \& des choses inouies que chacun de nous a tant raison d'esperer de toutes ses grandes qualitez ${ }^{51}$.

La fin du propos suggère une attente des hommes, un espoir placé en un roi jugé comme possédant toutes les vertus idéales de la fonction et qu'il se doit de conserver.

Sans être une mise en garde pour le roi, cette traduction et ses Remarques offrent un miroir pour rappeler ce que doit être, à ses yeux, la pratique du pouvoir : celle d'un père. Marolles développe en ce point une vision proprement française remontant à l'époque de Louis XII, qualifié de "Père du peuple " lors des États Généraux réunis à Tours en mai 1506. Il le mentionne explicitement dans la dédicace de $1649^{52}$ et développe cette position à plusieurs reprises dans le cadre de ses commentaires, tant pour la première traduction que pour la seconde. Ce regard d'homme du xvII ${ }^{e}$ siècle se retrouve dans l'absence d'une admiration sans faille de l'abbé pour les agissements d'Énée, dont il a pourtant repris le statut de prince vertueux. Lorsque le comportement $\mathrm{du}$ fils d'Anchise lui semble contestable au regard des conceptions modernes, il le signale explicitement, et plus particulièrement dans ses Remarques de 1662. Ainsi, au Livre II, le héros raconte-t-il les massacres perpétrés dans le palais de Priam et auxquels il a assisté ; Marolles se montre alors sévère :

499. Je veis Neoptoleme, \&c. On reprend icy Virgile, \& pour plusieurs vers qui sont en suitte de celui-cy, de ce qu'il fait raconter à Enée, ce qu'il vid dans le Palais de Priam, quand la porte en fut forcée par Neoptoléme fils d'Achile, \& on dit que ce n'est pas le representer bien valeureux de luy faire voir du haut d'une tour ce qui se passe en ce lieu-là, \& de ne s'exposer point luy-mesme dans le peril pour resister avec ses amis à la violence des Grecs : Et qu'enfin il ne luy est pas fort glorieux de voir tuër Polite son beau-frere, \& le Roy Priam son beau-pere, sans y faire de resistance : car en effect c'estoit une occasion où il falloit perir. J'avoüe, que cela est difficile à sauver, du moins de la façon que nous concevons aujourd'huy la valeur 
des Heros, \& qu'il a de l'apparence qu'elle se doive concevoir. Sans mentir, il y a bien de l'apparence que Virgile n'a pas considéré cecy d'assez prés, \& que pour orner sa narration d'une circonstance necessaire pour dépeindre la Catastrophe de l'Empire de l'Asie, il n'a pas assez observé ce qui estoit de la bienseance pour son Heros. Que diroit la dessus Jules Cesar Scaliger, si grand admirateur de Virgile ? Que diroient Servius, Donat, les doctes Jesuites Lacerda \& Pontanus, \& les autres Partisans les plus passionnez? Pour moy, je me rends \& je ne voudrois jamais entreprendre la deffense d'une cause qui n'est pas soutenable ${ }^{53}$.

L'abbé ne nomme pas les auteurs des critiques à l'encontre du héros latin, comme en témoigne l'usage du « on ", mais il prend position à titre personnel, se démarquant des commentateurs antérieurs admiratifs de Virgile. Son analyse s'explique par son état d'ecclésiastique qui confère un enjeu moral et politique à ses Remarques destinées au Roi comme au plus grand nombre.

Que Marolles s'empare de l'Énéide en raison du profit moral qu'un lecteur peut en tirer, ne doit pas toutefois effacer la dimension littéraire qu'il donne à son travail. Au fil de ses Remarques, Marolles utilise l'épopée de Virgile pour discuter des codifications contemporaines $\mathrm{du}$ genre épique. Les considérations sur le pouvoir royal sont l'occasion de rappeler les traits de caractère du héros épique et l'abbé étend ses commentaires au style propre à ce type de poèmes. Grâce à la distinction des styles utilisés, il présente les trois premiers vers de l'Énéide comme emblématiques du parcours poétique de Virgile: le premier renvoie aux Bucoliques, le deuxième aux Géorgiques et le troisième à l'Énéide ${ }^{54}$. Puis il développe ce que doit être à ses yeux le style héroïque :

4. Gratum opus Agricolis. Il parle des Géorgiques, qu'il appelle ouvrage agreable, pour les Laboureurs, où certainement le stile est plus relevé que dans les Bucoliques; mais il ne l'est pas tant aussi que dans l'Eneide. En quoi le Poëte fait paroistre son grand jugement, dans ces trois sortes d'Ouvrages, parce qu'en effet, le stile se doit hausser ou baisser, selon que les sujets l'exigent. Ce qu'il a partout observé admirablement. Or dans les matieres heroïques toutes les choses ne sont pas également sublimes. C'est pourquoy il faut qu'il y ait par tout une grande varitation, sans y perdre pourtant jamais la gravité ou la majesté du stile héroïque, lequel pour n'estre pas en tout lieu également sublime ou figuré, ne doit pourtant jamais ramper ny degenerer dans les impertinentes allusions des mots, ou les basses railleries ${ }^{55}$.

Nourri par ses traductions antérieures de genres variés ${ }^{56}$, Marolles développe en 1662 ce qui ne faisait l'objet que d'un bref commentaire en $1649^{57}$ et ses ajouts, dans lesquels il use des verbes « devoir » et "falloir », dévoilent le théoricien. Ce type de Remarques, disséminées au gré des différents vers, s'explique sans doute par la concomitance de son travail de commentateur et de théoricien puisqu'il joint à sa traduction de 1662 un Traité du poème du poème épique, comme nous l'avons dit précédemment. Il est l'occasion pour lui de célébrer l'elocutio virgilienne, dont il souligne la gravité, l'élégance et la concision, qualificatifs qui sont traditionnellement attribués à Virgile. Il n'en reste toutefois pas au stade de l'admiration : s'il place l'auteur latin en position de modèle générique supérieur à Homère, il tient à évoquer sa filiation littéraire et, surtout, sa postérité jusque dans les œuvres les plus contemporaines. Ce dessein, plus novateur, se perçoit dans le traitement qu'il accorde aux comparaisons dans ses commentaires de 1662, traitement qui explique leur étendue considérable par rapport à ceux de 1649 . Pour cela, Marolles s'appuie largement sur le chapitre XIV du cinquième livre de la Poétique de Jules César Scaliger consacré aux comparaisons ${ }^{58}$. Il cite les différents auteurs, latins ou grecs, qui ont usé d'une comparaison similaire, mentionne la référence précise, transcrit le texte antique (à l'exception des textes grecs) et en 
propose presque systématiquement la traduction, qui provient pour beaucoup de ses travaux antérieurs. Il complète ce qui nous semble relever de l'emprunt ${ }^{59}$ par des jugements esthétiques qui ne s'accordent pas toujours avec ceux de Scaliger, et augmente son travail d'une recherche du même ordre dans les épopées des $\mathrm{XVI}^{\mathrm{e}}$ et $\mathrm{XVII}^{\mathrm{e}}$ siècles dont il donne les passages correspondants. À propos de la comparaison avec le serpent, il fait ainsi figurer des extraits de La Jérusalem délivrée du Tasse (1581), du Moïse sauvé de Saint Amant (1653), du Saint Louis, ou la Sainte couronne reconquise sur les infidèles du Père Le Moyne (1653), de La Pucelle, ou la France délivrée de Chapelain (1656), du Clovis ou la France chrétienne ${ }^{60}$ de Desmarest de Saint Sorlin (1657) et l'on trouve cités à plusieurs reprises pour d'autres comparaisons la Franciade de Ronsard (1572), le Saint Paul d'Antoine Godeau (1654) et le poème sur Jésus Christ d'Arnaud d'Andilly ${ }^{61}$. Dépassant la simple compilation, il n'hésite pas à souligner la beauté de ces passages qui ne le cèdent en rien, à ses yeux, à leur source, voire l'emportent sur elle.

Cette filiation antique et cette postérité épique de l'Énéide sont établies en termes d'imitation par Marolles. Mais celui-ci élargit son champ d'investigation en insérant dans ses Remarques des œuvres appartenant à d'autres genres que l'épopée. Il parle notamment de l'influence exercée par l'Énéide sur l'embrasement de Troie décrit par Pétrone dans le Satyricon ${ }^{62}$ et cite dès sa Préface de 1649 le Virgile travesti de Scarron mais aussi les tragédies de Didon se sacrifiant de Jodelle (1558) et de Hardy (1603), et la Didon de Scudéry (1637) à propos du chant IV de l'Énéide ${ }^{63}$. Marolles aborde ainsi, quoique de façon implicite, la question du rapport au modèle dans le processus de création littéraire. Il définit ce rapport comme une imitation plus ou moins proche, comme la reprise d'un motif, - indépendamment du genre-, ce dont témoigne l'expression "avoir beaucoup de rapport à » à propos d'un passage de Lucain qui puise chez Ovide pour évoquer la tempête ${ }^{64}$. L'apparition du terme d'« émulation", bien qu'appliqué à des auteurs antiques ${ }^{65}$, vient alors qualifier la relation des auteurs avec leurs sources. Ce mot nous semble également inscrire l'ample travail théorique et critique de Marolles dans une perspective plus ambitieuse que la simple restitution et intelligence d'un texte antique. Virgile est certes un modèle incontesté pour le genre épique, mais l'éloge que fait Marolles de ses propres contemporains, tout comme ses considérations sur la langue française, sont une invitation, consciente ou non, pour les lettrés du XviI ${ }^{e}$ siècle à s'inscrire dans une démarche de concurrence stimulante et d'émancipation ${ }^{66}$ vis-à-vis de la source antique, et partant, une invitation à créer des modèles français d'un égal prestige, voire d'un prestige supérieur.

Ce dialogue littéraire à propos de la définition d'une épopée à la française s'inscrit dans l'entreprise plus vaste de promotion de la langue et de la prose française. Dans ses commentaires et ses écrits liminaires, l'abbé insiste sur les potentialités de celle-ci, et ce dès 1649 :

[...] je me suis persuadé que nostre prose estoit capable aussi bien que celle des Grecs \& des Latins, de soustenir quelquefois les graces de la Poësie, quand on s'y veut soigneusement appliquer ${ }^{67}$.

Sa traduction en prose se met donc au service de cette promotion et plusieurs Remarques tendent à souligner la capacité de la prose à restituer une écriture poétique dont il n'a eu de cesse de vanter les qualités, souhaitant ainsi rehausser davantage le français. Marolles revendique alors une prose poétique, notamment lorsqu'il justifie l'emploi de certaines figures dans le «style ordinaire » face aux critiques de certains :

Ceux qui tiennent que la Poësie consiste seulement en des lignes mesurées, ou en certains nombres de syllabes, qui font une harmonie agreable, reprendront possible 
quelques expressions dont je me suis servi, pour n'estre pas assez du stile ordinaire, ou pour estre trop Poëtiques, quoy que je traduise un Poëte. [...] Mais comme il ne seroit pas mal-aisé de monstrer qu'ils se peuvent tromper, ou qu'ils ont perdu le souvenir de ces charmantes Poësies en prose, \& du stile Poëtique de Xenophon, de Platon, de Philostrate, de Lucien, d'Achilles Statius, de Pline le jeune, de Petrone, d'Apulée, \& d'une infinité d'autres entre les anciens, aussi bien que des façons de parler figurées de nostre Balzac et de nos Traducteurs des Metamorphoses d'Ovide, de la Jerusalem du Tasse, du fameux Roland de l'Arioste, de l'Histoire Ethiopique d'Heliodore, des Couches de la Vierge de Sannazare, \& de tant d'autres ouvrages agreables, qui sont donnez par la liberalité de nos Muses aux divertissements de la Cour ; il me suffira de dire qu'on l'a fait à dessein, \& qu'il seroit de mauvaise grace de traduire un Poëte comme un Historien ${ }^{68}$.

Mais le visage qu'il donne à cette prose est celui du début du $\mathrm{XVII}^{\mathrm{e}}$ siècle, et sa promotion est surannée pour la traduction vers $1650^{69}$. Ainsi s'expliquent sans doute le petit nombre de commentaires de ce type dans sa traduction de 1662 et la disparition du panégyrique d'une telle écriture dans la Préface ${ }^{70}$. Si Marolles se tourne vers les réflexions génériques (nous l'avons vu), il n'en demeure pas moins que ses prises de position transforment l'espace des notes finales en lieu de dialogue; l'œuvre antique est devenue le truchement par lequel s'entame cet échange, fragmenté au gré des vers qui ont retenu son attention, plurielle dans ses sujets et ses enjeux, tant moraux que littéraires.

Se tourner vers Virgile pouvait passer pour le geste d'un Ancien et le traduire en prose dans la deuxième moitié $d u X \mathrm{XII}^{\mathrm{e}}$ siècle pour une entreprise passéiste. Pourtant, le travail de Marolles dans ses Remarques témoigne d'une démarche plus originale et moderne qu'il n'y paraît. Héritier des commentateurs des siècles passés, Marolles reprend à son compte une partie de leurs remarques qu'il destine au plus grand nombre dans un souci de vulgarisation; celle-ci passe par le recours à la traduction en français, tant pour le texte source que pour les observations. Cet apparat critique, où le plaisir d'une certaine érudition transparaît, doit servir, avant toute chose, à l'« intelligence facile ${ }^{71}$ » de la lettre du texte. Mais il contribue également à la lisibilité $\mathrm{du}$ texte pour un lectorat $\mathrm{du}$ XVII ${ }^{\mathrm{e}}$ siècle. Ce glissement de la restitution à la recevabilité d'une œuvre antique entraîne l'abbé vers des observations plus personnelles. Quittant sa position de passeur entre deux époques, Marolles place l'œuvre entre lui et ses contemporains $\mathrm{du} \mathrm{XVII}^{\mathrm{e}}$ siècle en vue d'une réflexion morale, politique et littéraire. S'il fait toujours d'Énée le parangon du prince vertueux, c'est pour mieux définir ce que doit être la pratique du pouvoir royal et s'adresser au jeune Louis XIV; s'il inscrit l' Énéide dans un héritage antique et une postérité contemporaine, c'est pour donner à voir la translatio de l'œuvre virgilienne. Il transmet à son lecteur une démarche de création littéraire, pensée en termes d'émulation créatrice, et il lui propose un discours théorique moderne sur l'épopée (quoique fragmentaire) ; son travail devient une sorte d'invitation implicite au dépassement du modèle antique vers la création d'une œuvre conforme aux attentes esthétiques $\mathrm{du}$ XVII ${ }^{\mathrm{e}}$ siècle, et digne d'être un modèle. Quand il se tourne vers Virgile, geste digne d'un Ancien, Marolles regarde, en Moderne, vers sa propre époque, et cela, même si sa vision de la prose est déjà vieillie. 


\section{NOTES}

1. Michel de Marolles publie une première traduction de Lucain en 1623, qu'il reprend en 1647 et 1649.

2. Les CEuvres de Virgile, traduites en prose, enrichies de figures, tables, Remarques, Commentaires, éloges et Vie de l'Autheur, Avec une explication geographique du voyage d'Enée, \& de l'ancienne Italie, et un abbregé de l'histoire divisé en trois livres, contenant ce qui s'est passé de plus memorable depuis l'embrazement de Troye, jusques à l'Empire d'Auguste, pour l'intelligence du Poëte, Au Roy, par Michel de Marolles, Abbé de Villeloin, A Paris, Chez Toussaint Quinet, au Palais sous la montée de la Cour des Aydes, MDCXLIX, Avec Privilège du Roy. L'ouvrage dédié au roi, de format in quarto, propose, pour l'Énéide, 73 pages de Remarques finales, présentées par chant suivant l'ordre du texte. Il ne comporte pas le texte latin. L'ouvrage ne comprend qu'un seul volume divisé en deux parties. La première est consacrée à l'Énéide, la seconde aux Bucoliques et aux Géorgiques. Cette dernière est marquée par une nouvelle pagination.

3. L'Énéide de Virgile, en latin et en françois, avec des Remarques utiles \& fort amples, accompagnées d'un Traité du Poëme Epique ; pour montrer de quelle sorte celui-cy a esté imité des Grecs, \& comme l'ont aussi imité plusieurs Escrivains Celebres de diverses Nations, qui sont venus depuis, Par M. de Marolles, abbé de Villeloin, A Paris, Chez Guillaume de Luyne, Libraire Juré, au Palais, en la Gallerie des Merciers, à la Justice, MDC LXII, 3 vol. L'ouvrage, in octavo, également dédié au roi mais présentant le texte latin en regard de la traduction française, offre 703 pages de Remarques sur les différents vers de l'Énéide, présentées suivant l'ordre des vers dans chaque chant à la fin du premier et du deuxième tome. Le troisième tome est consacré aux Bucoliques et aux Géorgiques.

4. Toutes les œuvres de Virgile traduites en vers françois. Divisees en deux Parties. Dediees au Roy, Seconde Partie contenant les douze Livres de l'Eneide avec des Remarques \& des Tables tres-amples, A Paris, Chez Jacques Langlois, Imprimeur ordinaire du Roy, dans la Grand'Salle du Palais, au sixiéme Pillier, vis-à-vis la Grand'Chambre, à la Reyne de Paix ; et Emmannuel Langlois, ruë Saint Jacques, à la Reyne du Clergé, M.DC.LXXIII, avec privilege du Roy.

5. L'Énéide de Virgile, traduit en vers par M.D.A.D.V., A Paris, MDCLXXI, avec privilège du Roy. Au verso de la page de titre se trouve décrit le contenu de l'ouvrage : «Ce recueil contient quelques Pieces du premier \& du troisiéme Livres de l'Eneide, dispersées en divers lieux, Avec 109 Comparaisons que ce Poëte a employées dans tout son Ouvrage. A quoy ont esté adjoustées les Traductions en Vers des IV. VI. VII. VIII. IX. \& X. Livres, Et encore plusieurs Epistres, Discours \& Prefaces pour servir d'ornement \& d'instruction sur ce sujet. " La pagination de l'ouvrage est continue, et chaque traduction, où ne figure pas le texte latin, est précédée d'une page de titre, d'une dédicace et d'une préface. L'absence de mention d'un imprimeur libraire sur les pages de titre peut laisser supposer qu'il s'agit d'une impression à frais d'auteur regroupant les étapes intermédiaires d'un travail en cours.

6. Marolles mentionne dans l'« Avis au Lecteur» de la traduction inachevée du Livre IV (ibid., p. 462) : «Et pendant qu'on a fait l'Edition de ce 4. Livre, je ne sçaurois m'empescher de dire icy que j'ay achevé la Traduction du 5. Livre, qui n'est pas une petite avance du reste de l'Ouvrage, où je me propose de travailler, sans oublier les Bucoliques, afin de ne rien obmettre dans nos Vers, apres ceux de Virgile, de tout ce qui nous reste des CEuvres d'un si excellent Poëte. »

7. Joachim Du Bellay, Le quatriéme livre de l'Eneïde de Virgile, traduict en vers François, La Complainte de Didon à Enée, prince d'Ovide ; \& d'autres œuvres de l'invention du Translateur, par J.D.B.A. [Joachim Du Bellay, Angevin], à Paris, par Vincent Certenas, 1552 ; et id., Deux livres de l'Éneide de Virgile, a scavoir le quatrieme, et sixieme, traduicts en vers francois par J. Du Bellay, angevin avec la Complainte de Didon à Enee, prise d'Ovide, la Mort de Palinure, du cinquieme de l'Éneide,\& l'Adieu aux Muses, pris du latin 
de Buccanan, A Paris, de l'imprimerie de Federic Morel, rue S. Jan de Beauvais, au franc Meurier. M. D. LX. Avec privilege.

8. Jean Davy du Perron, Partie du premier livre de l'Æneïde de Virgile, Paris, Robert Etienne, 1610 ; l'ouvrage est en vers et sans nom, mais l'auteur en est Jean Davy du Perron, cardinal et évêque d'Évreux; et id., Partie du premier et quatriesme livre de l'Ænéide de Virgile, Paris, Robert Etienne, 1611.

9. Jean Bertaud, Traduction du second livre de l'Enéïde, en vers françois, par Jean Bertaud, Evêque de Sées, dans Les Euvres de Mademoiselle de Gournay et dans le recueil de Poësies de M. Bertaud, Paris, Toussaint du Bray, 1620.

10. Marie de Gournay, Versions de quelques pièces de Virgile, Tacite et Saluste, avec l'institution de Monseigneur, frère unique du roy... par la damoiselle de Gournay [et M. Bertault, évêque de Séez. Précédé d' un traité sur la poésie, par Mlle de Gournay], Paris, F. Bourriquant, 1619.

11. Pierre Perrin, L'Eneïde, traduite en vers François : premiere partie, contenant les six premiers livres, dédiée à l'Eminentissime Cardinal Mazarin, avec des remarques du Traducteur aux marges, pour l'intelligence de la carte, \& de l'histoire ancienne, veritable \& fabuleuse, par Pierre Perrin, Conseiller du Roi en ses Conseils, Introducteur des Ambassadeurs \& Princes étrangers près la personne de son Altesse Royale M. le Duc d'Orleans, Paris, Pierre Moreau, seul Imprimeur \& Graveur ordinaire du Roi de la nouvelle imprimerie, 1648.

12. Annibal Caro, L'Eneide di Virgilio, del commendatore Annibal Caro, Venise, B. Giunti et frères, 1581.

13. Octavien de Saint Gelais, Les Eneydes de Virgile, translatez de Latin en François, par Messire Octavian de Sainct Gelais, en son vivant Evesque d'Angoulesme, revuës \& cottés par Maître Jehan Divry, Bachelier en Medecine, Paris, Antoine Verard, 1509. L'ouvrage connaît une nouvelle édition en 1529 et en 1540 .

14. Louis des Mazures, L'Eneïde de Virgile, Prince des Poëtes Latins, translatée de Latin en François, par Loüis des Mazures, Tournisien, Lyon, Jean de Tournes, 1560.

15. Robert et Antoine Le Chevalier d'Agneaux, Les Euvres de Virgile Maron, traduites de Latin en vers françois, par Robert et Antoine Le Chevalier d'Agneaux, freres, de Vire en Normandie, dédiée au Roi, Paris, Thomas Périer, 1584 (une deuxième édition paraît en 1583 chez Guillaume Auvray avec le texte latin en marge, puis une troisième, toujours avec le texte latin en marge, chez David Le Clerc, en 1607).

16. René de La Mothe du Tertre, L'Eneïde de Virgile en prose Françoise ; savoir, les six premiers livres, \& les autres par M. Du Pelliel, avec enrichissemens de figures, Paris, Toussaint du Bray, 1626.

17. L'Eneïde de Virgile, traduction nouvelle, par le sieur Tournay, Paris, Antoine de Sommaville, 1648.

18. Pierre Perrin, L'Eneide, seconde partie, contenant les six derniers livres, dédiée à Monsieur le Cardinal Barberin, fidellement traduite en vers héroïques, avec le Latin à côté, \& les remarques à chaque livre, pour l'intelligence de l'histoire \& de la fable, enrichie de figures en taille douce, Paris, Etienne Loyson, 1658.

19. Marolles, Les Euvres de Virgile (1649), op. cit., préface non pag.

20. Ibid.

21. Id., L'Énéide de Virgile (1662), op. cit., préf. non pag.

22. Cette perspective s'inscrit dans les débats sur la traduction qui agitent le milieu du siècle et dont Port-Royal est l'un des foyers. Marolles fut en contact proche avec les milieux de Port-Royal. Pour plus de détails sur la position de Marolles dans ce débat, on se reportera à nos analyses dans Sénèque le Tragique en France (XVI-XVII siècles), Paris, Classiques Garnier, 2011, p. 733-745.

23. Marolles, Les CEuvres de Virgile (1649), op. cit., préf. non pag.

24. Id., L'Énéide de Virgile (1662), op. cit., vol. 1, préf. non pag.

25. Voir ibid.., vol. 1, p. 257-258: «Plusieurs en [des remarques] ont écrit devant moy, sous le nom de Commentaires, d'Annotations \& d'Observations; les uns anciens, comme Servius Honoratus, Donat, Pomponius Sabinus, le Grammairien Probus, Philargyrius, Modestinus. Les autres modernes, tels que Germanus Valens, Adrian Turnebus, Louys Lucreda [sic pour Lacerda], 
Jacques Pontanus, Hortensius Montfortiensis, Nascimbenus, Nannius, à Myen, Abramus, Jodocus Badius Ascentius, Philippe Beroalde, Philippe Melancton, Mancinellus, Domitius Calderinus, Augustinus Dathus, Pierius Valerianus, Janus Parrasius, Janus Guilielmus, Baptista Pius, Robortellus, Alexandre de Naples, Chrystophorus Landinus, Joannes Hartungus, Jodocus Vvillichius, Camerarius, Georgius Fabricius, Corrardus, Erythreus, de Bonfinis, Jean Louys Vives, Adrianus Burlandus, Casaubon, Lipse, Marc-Antoine Muret, Scaliger, Lambin, Gifarius, Taubmannus, Claude Saulmaise, Ludovicus Lucius Professeur de Basle, Magius, Merula, Thomas Farnabius, \& Cornelius Schrevelius. » Voir infra note 48 : en 1649, mentionnant les commentaires de Pontanus et La Cerda, Marolles ajoutait ceux d'un autre jésuite, «Gallucius Sabinus », non repris ici (Les CEuvres de Virgile [1649], op.cit., préf. non pag.) - Tarquinio Galluzzi, Virgilianae Vindicationes..., Rome, A. Zannetti, 1621. Voir aussi, dans l'Atelier de la présente livraison, la liste des commentateurs que donne Jacob Pontanus (qui cite sans coquille le nom de Corradus).

26. Ibid., préf. non pag.

27. Ibid., p. 257.

28. Ibid., vol. 1, préf. non pag.

29. Ibid.

30. Id., Les CEuvres de Virgile (1649), op. cit., p. 424.

31. Ibid., p. 418. La remarque est identique dans l'édition de 1662 (op.cit., vol. 2, p. 403). Nous traduisons : «Il faut assurément reconnaître que ce lieu fait partie de ceux qui, dans les douze livres de Virgile, sont obscurs par nature, ou impossibles à résoudre, ou à corriger, ou laissés tels quels sans que nous puissions les comprendre avec certitude faute de connaître l'histoire antique."

32. Id., Les CEuvres de Virgile (1649), op. cit., p. 395-396. Nous mettons ci-après les commentaires de l'édition de 1662 qui ne diffèrent légèrement que dans la formulation de la dernière phrase (id., L'Énéide de Virgile (1662), vol. 1, op. cit., v. 545, p. 646) : «545. Je suis prest de m'en aller, \& d'achever le nombre.] Il y a au Latin, explebo numerum. Ce qui est mal-aisé à entendre, \& souffre beaucoup d'interprétations. Servius l'explique, je diminueray le nombre, voulant dire qu'il se retireroit de la compagnie d'Enée et de la Sibylle. Mais ceux que j'ay suivis l'entendent, ou du nombre des années qu'il eust deu vivre en ce monde, ou de celles qu'il devoit passer en l'autre dans le lieu de purgation, avant que d'être admis dans le sejour des bienheureux. "

33. Id., Les CEuvres de Virgile (1649), op.cit., p. 418 et id., L'Énéide de Virgile (1662), vol. 2, op.cit., p. 401.

34. Nous aurons l'occasion de revenir sur les comparaisons ci-après, en raison du traitement qu'il leur consacre dans l'édition de 1662 et qui s'inscrit également dans une intention autre que pédagogique.

35. Id., Les Euvres de Virgile (1649), op. cit., p. 407.

36. Nous traduisons : «L'onde s'étonne d'offrir un refuge à la guerre et s'éclaire du reflet brillant des armes ».

37. Marolles, L'Énéide de Virgile (1662), vol. 2, op. cit., p. 349. Il s'agit d'un ablatif absolu : « les flots de la mer s'étonnant ».

38. Id., Les CEuvres de Virgile (1649), op. cit., p. 418 et id., L'Énéide de Virgile (1662), vol. 2, op. cit., p. 409.

39. Id., Les Euvres de Virgile (1649), op. cit., p. 406 et id., L'Énéide de Virgile (1662), vol. 2, op. cit., p. 51.

40. Id., Les CEuvres de Virgile (1649), op. cit., p. 177-179.

41. Id., Les CEuvres de Virgile (1649), op. cit., p. 180-208.

42. Id., Les Cuvres de Virgile (1649), op. cit., p. 407.

43. Id., L'Énéide de Virgile (1662), vol. 2, op. cit., p. 348-349.

44. En tant que passeur, et passeur pédagogue, il se place en intermédiaire entre ses contemporains et l'Antiquité. 
45. Id., Les CEuvres de Virgile (1649), op. cit., p. 366. Le vers de Virgile est: « Non haec sine numine diuom / eueniunt » (trad. Jacques Perret : « Ces choses n'arrivent pas sans le vouloir des dieux »).

46. Id., L'Énéide de Virgile (1662), vol. 1, op. cit., p. 402.

47. Id., Les CEuvres de Virgile (1649), op. cit., préf. non pag. Pour se justifier, Marolles s'appuie sur des arguments d'autorité dans la suite du passage et énumère un grand nombre d'ecclésiastiques ayant traduit des œuvres profanes (ibid.) : «Et s'il faut des exemples de ce que j'ay fait, outre Monsieur le Cardinal du Perron, \& les Evesques d'Angoulesme [Octavien de Saint Gelais] \& de Sées [Jean Bertaud], que j'ay desja nommez, ne sçavons nous pas que l'Evesque Eustatius a fait un tres-ample Commentaire sur toutes les Poësies d'Homere? Heliodore n'est-il pas autheur de l'Histoire des amours de Theagene \& de Cariclée, que M. Jacques Amiot E. d'Auxerre a traduite, aussi bien que les Euvres de Plutarque, de Diodore Sicilien, \& le Livre des amours de Daphnis \& de Cloé ? Jerosme Vidas E. d'Albe a composé des Poëmes du ver à soye, \& du jeu des Echets. J'ai appris de M. de Salmonnet, qui joint une grande erudition à une extreme courtoisie, que Gravinus Douglasius E. de Dunkelle en Escosse dans la province de Perth a fait il y a plus de six vingts ans, une traduction en vers de l'Eneide de Virgile, en sorte que chaque vers Escossois respond à chaque vers Latin. M. Coeffeteau E. de Dardanie, \& depuis E. de Marseille, de l'Ordre de S. Dominique, a traduit le Florus, \& a composé un abregé du Roman d'Argenis. Claude de Seissel Arch. de Turin, a traduit l'Histoire de Thucydide, \& plusieurs autres pieces semblables. Messire Philippes de Vitry E. de Meaux, escrivit en vers de son temps une Paraphrase des Metamorphoses d'Ovide, en faveur de la Reine Jeanne, dont le Manuscript est curieusement gardé en la Bibliotheque de S. Victor à Paris, \& mourut en l'année 1351; Et nous voyons par les Commentaires de Jacobus Pontanus, de Ludovicus Lacerda, de Gallucius Sabinus, sur Virgile ; \& par ceux de Raderus, \& de Martin Delrio sur Martial, \& sur Seneque, comme les Peres Jesuites, qui sont d'une Compagnie de grande reputation pour la pieté, se sont exercez bien souvent sur un pareil sujet."

48. Commentant la première comparaison de l'Énéide en 1649, Marolles ajoute (ibid., p. 350) : « De cette comparaison qui est la premiere de ce grand ouvrage, les Seigneurs \& tous ceux qui ont authorité sur le peuple doivent apprendre à se rendre recommendables par la vertu. »

49. Ibid. « Au Roy », non pag.

50. Ibid.

51. Id., L'Énéide de Virgile (1662), op. cit., « Au Roy », non pag.

52. Id., Les CEuvres de Virgile (1649), op. cit., « Au Roy », non pag. : " Virgile l'appelle Pere, pour dire le Prince Énée. Car, SIRE, les Princes \& les Roys, comme celuy-cy, doivent tenir lieu de Peres de leurs peuples \& leur dignité, sans doute, est une dignité de Pere \& une charge de Pasteur. Ce que sçeut si bien reconnoistre un des predecesseurs de vostre Majesté, \& du mesme nom qu'elle porte, qu'il se glorifia du titre que les Estats assemblez lui donnerent de Pere du Peuple en la ville de Tours. »

53. Marolles, L'Énéide de Virgile (1662), op. cit., vol. 1, p. 390-391. Le terme de bienséance renvoie certes à la convenance du caractère d'Énée sur le plan littéraire, mais cette convenance repose sur une conception du héros épique propre au XVII ${ }^{\mathrm{e}}$ siècle, tant sur le plan moral que littéraire.

54. Ibid., p. 363 : « Les trois stiles s'y observent en peu de paroles, l'humble dans le premier Vers, le mediocre dans le second, \& le grand dans le troisieme pour marquer les Ouvrages qu'il avoit faits. "

55. Ibid., p. 265.

56. Il a achevé la traduction des tragédies de Sénèque, qui se caractérise par un style "sublime », des comédies de Plaute et de Térence : l'expression «basses railleries » peut faire allusion à Plaute.

57. Id., Les Cuvres de Virgile (1649), op. cit., p. 345 : «Ouvrage agréable au peuple rustique.] Cela s'entend des Géorgiques, où le stile est plus relevé que dans les Bucoliques, \& ne l'est pas tant que dans l'Eneide. » 
58. Jules César Scaliger, Julii Caesaris Scaligeri, viri clarissimi, Poetices libri septem. Ad Sylvium filium, apud Antonium Vincentium, M.D.LXVI. Le livre V a été traduit par Jacques Chomarat : La Poétique, V, Le Critique, Genève, Droz, 1994.

59. Il conviendrait de mener une comparaison approfondie de l'ouvrage de Scaliger et du travail effectué par Marolles. L'ampleur d'une telle investigation ne pouvait trouver sa place dans les limites de cet article.

60. Marolles, L'Énéide de Virgile (1662), op. cit., vol. 1, p. 383-387.

61. On ne peut qu'être frappé par la quasi-exclusivité des sujets religieux des poèmes cités. La question de la présence du merveilleux chrétien dans le genre épique sera une dizaine d'années plus tard au cœur de la querelle des Anciens et des Modernes. Marolles ne semble pas initier de débat sur ce point dans ses Remarques de 1649 et 1662. L'étude des Remarques de sa traduction en vers de 1673 (que nous n'avons pas prise en compte pour le présent article) permettrait de s'interroger sur cet aspect.

62. Id., L'Énéide de Virgile (1662), op. cit., vol. 1, p. 354.

63. Id., Les CEuvres de Virgile (1649), p. 673 : «Plusieurs endroits de ce mesme livre, qui ont aussi esté imitez par M. de Scudery dans sa Tragedie de Didon, ont encore servy de matiere à l'une et l'autre Didon se sacrifiant d'Estienne \& d'Alexandre Hardy, qui ont esté des Poëtes celebres en leur temps. »

64. Id., L'Énéide de Virgile (1662), op. cit., vol. 1, p. 291.

65. Id., L'Énéide de Virgile (1662), op. cit., vol. 1, p. 354. Marolles cite des vers de Pétrone, tirés de «son Poëme de l'embrazement de Troye, lesquels sont tout à fait élegants, \& qu'il est aisé de juger qu'il les auoit faits par émulation de ceux de Virgile».

66. S'émanciper ne doit pas être entendu ici en termes de rejet, mais bien de dépassement et de filiation prenant en considération les critères esthétiques du XVII ${ }^{\mathrm{e}}$ siècle.

67. Marolles, Les CEuvres de Virgile (1649), op. cit., préf. non pag.

68. Ibid.

69. Nous renvoyons sur ce point aux analyses de R. Zuber dans Les Belles infidèles et la formation du goût classique, Paris, Albin Michel, 1990 (plus particulièrement à la première partie de l'ouvrage, aux chapitres IV et V, p. 97-161).

70. Marolles a en effet célébré l'éloquence de la chaire, du parlement, la beauté des traités de morale ; mais il a également souligné la grandeur de ceux qui écrivent en vers dans une sorte d'élan vigoureux pour une défense plus générale des beautés de la langue française.

71. L'Énéide de Virgile (1662), op. cit., vol. 1, préf. non pag.

\section{AUTEUR}

\section{FLORENCE DE CAIGNY}

CELLF $17^{\mathrm{e}}-18^{\mathrm{e}}$, UMR 8599 